\title{
General Surgery during Pandemic Era - Evolving Strategies: A Cohort Study from a Tertiary Care Centre in North India
}

Keywords: COVID-19; Pandemic; Aerosol Generating Procedures Laparoscopic Surgery; General Surgery

\section{Abstract}

Due to Corona Virus disease 2019 (CoViD-19) pandemic healthcare systems worldwide have been suffering in terms of their capability to manage affected people and the ability to provide standard treatment. Multiple vaccines have been developed and it is being implemented globally on a large scale. However, mutant strains of the virus are being detected from all parts of the world and pandemic and it's associated concerns are continuing. With the majority of patients being asymptomatic and high infectivity, safety related concerns have been there both for patients as well as healthcare workers. Various academic associations have issued guidelines to address these issues. The authors aim to provide a comprehensive overview of essential measures that healthcare providers and surgeons adopted to ensure safe performance of both elective and emergency surgical procedures at their institute.

Total of 506 patients underwent 512 general surgical elective and emergency procedures between 1st April 2020 to 31st December 2020 for different indications. The surgeries included both laparoscopic and open approaches. The strategies for preoperative testing for the presence of SARS $\mathrm{nCov} 2$ have been varying with availability of more evidence and techniques. For the initial six months, it was primarily R PCR for both elective and emergency cases. However, in the last three months, the majority of the patients were tested for SARS nCov2 via R PCR and emergency cases who were tested by CBNAAT Gene-Xpert.

The pandemic has affected the patient care globally. Various guidelines have been issued by different academic associations. However, every institution has to improvise depending upon the local resources and infrastructure available. With continuing pandemic every institution shall require infrastructural changes to continue to deal with continuous inflow of infected patients.

\section{Introduction}

In late December 2019, outbreak pneumonia of unknown cause occurred in Wuhan, Hubei Province, China. [1] The underlying causative agent of this pneumonia wasidentified as a novel coronavirus, which was initially named as severe acute respiratory syndrome coronavirus 2 (SARS-nCoV-2). The World Health Organization named this disease as CoViD-19 (Corona virus disease-2019). On March 11, 2020, WHO had declared COVID-19 as pandemic disease and by $31^{\text {st }}$ December 2020, COVID-19 had spread to nearly 210 countries and territories worldwide with approximately more than 82 million cases and approximately 1.8 million deaths. [2] India has also been affected adversely with more than 10 million infected cases and 148,738 death by $31^{\text {st }}$ December 2020[3]. Healthcare workers have also suffered in high number with 7 times more risk than general

\section{Journal of} Surgery

\author{
Verma $\mathbf{N}^{1}$, Sehgal $\mathrm{L}^{2}$, Wadhawan $\mathbf{R}^{3}$, Minhas $\mathrm{V}^{4}$ and \\ Gupta $\mathbf{M}^{5}$
}

${ }^{1}$ Department of Minimal Access, GI \& Bariatric Surgery, HCMCT Manipal Hospital, India

${ }^{2}$ Department of General and Liver Transplant Anesthesia, Liver Critical Care, HCMCT Manipal Hospital, India

${ }^{3}$ Department of Minimal Access, GI \& Bariatric Surgery, HCMCT Manipal Hospital, India

${ }^{4}$ Department of General and Liver Transplant Anesthesia, HCMCT Manipal Hospital, India

5epartment of Minimal Access, GI \& Bariatric Surgery, HCMCT Manipal Hospital, India

\section{*Address for Correspondence}

Wadhawan R Consultant and Head Department of Minimal Access, Bariatric and GI Surgery HCMCT Manipal Hospital Sector 6, Dwarka, New Delhi110075,Tel: 91-9810155826 India; Email: randeepwadhawan@yahoo.com

Submission: 19 April, 2021

Accepted: 25 May, 2021

Published: 31 May, 2021

Copyright: () 2021 Verma N. This is an open access article distributed under the Creative Commons Attribution License, which permits unrestricted use, distribution, and reproduction in any medium, provided the original work is properly cited.

population.

The situation related to pandemic has been ever evolving over last one year. While there have been worries related to detection of mutant stains with high infectivity and mortality. On the other hand, the reports of development of multiple vaccines and implementation of vaccination drive are encouraging [4].

The virus has been isolated in virtually all body fluids \& tissues. Various procedures performed in operation theatre, like; airway management, cautery use etc. have been associated with increased generation of aerosol. Additionally, majority of these patients are asymptomatic [5]. Further, the morbidity and mortality increase significantly in a CoViD-19 positive patient undergoing elective surgery $[5,6]$. Hence, the caution has been issued from the beginning of pandemic regarding performance of both elective and emergency procedures.

The authors have reviewed their data of patients undergoing general surgical procedures over nine months (April 1, 2020 till December 31, 2020). Along with that the authors have also reviewed the process developing and modifying standard operating procedures (SOP) to ensure safety of patients and healthcare workers and thereby minimising the risk of exposure.

\section{Methods}

\section{Development of SOPs}

Based upon the guidelines and literature available, the SOPs were developed in March 2020 according to available infrastructure and resources with keeping patient and staff safety at the centre. They were updated again in May and September 2020 as per more knowledge and resources available and change in government policies and recommendations on testing and conduct of procedures. 


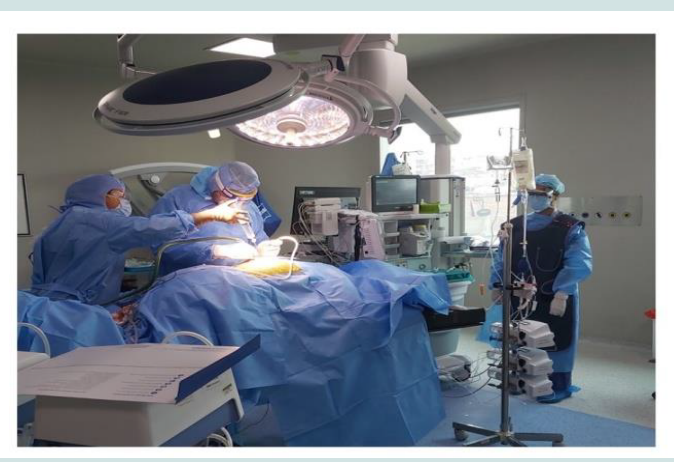

Figure 1: PPE for patient being operated after testing negative by RT-PCR.

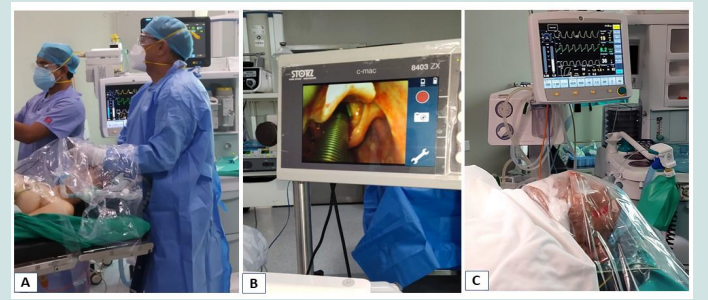

Figure 2: A-Transparent sheet covering during video laryngoscopy. B- Video laryngoscopy view of larynx. C - Transparent sheet covering over patient's face during an ongoing procedure.

Outpatient Management:The institution prioritized urgent or emergency visits and procedures. A separate triage area or fever clinic was created to screen for respiratory symptoms in any surgical patient visiting outpatient (OPD) or emergency (ER) department. Level 3 $\mathrm{PPE}$ is being used in ER and Flu clinic. The social distancing between doctors and patients is being maintained except during examinations. Only the most essential elements of the physical examination are conducted to minimize risk of transmission. Many patients are being assessed initially through video consultation. In OPD as well, due precautions with PPE are observed (hand hygiene, N95 mask, face shield or goggles).

Inpatient Management:For the first six months, all patients undergoing surgical procedure were tested for SARS nCov-2 by RTPCR assay. The in-patient department has been divided into three zones; green, amber and red. The patient admitted for elective surgery are admitted in amber zone first till their report was available. They are transferred to green zone once test report of negative is available and then proceed for surgery. In case of positive report or symptoms highly suggestive of CoViD-19, the elective surgical procedures are postponed.

In Emergency situations, if the surgery is not life or limb saving, and the procedure can be delayed for 24 hours, the patients are initially assessed for presence or absence of CoViD-19 clinically along with testing of nasopharyngeal swab for SARS nCov2. In case of life or limb saving surgeries, the sample is sent. However, required surgical procedure is performed in operation theatre designated for COVID positive patient taking all due precautions including donning of level-3 PPE.
However, in last three months, the patients requiring emergency procedures have been tested with CBNAAT (Cartridge Based Nucleic Acid Amplification Test) Gene-Xpert. Till the availability of the report, patients are being in an amber zone in emergency area. And depending upon the test results, they are being transferred to Green or red zone. For elective procedures, all patients are being encouraged to get tested with RT-PCR within 24-72 hours of planned procedure before admission. Depending on the positive or negative report, the procedures are being performed or deferred, respectively.

$\mathrm{X}$-ray of chest is routinely done for all patients. In case of emergency situation and high suspicion, the patients are evaluated with CT scan of chest.

Operation Theatre Management: The authors' institution does not have negative pressure OT for management of patient positive or suspected for COVID. Hence, an OT has been designated towards one end of OT complex along with dedicated donning and doffing area for PPE. A second OT close by has been identified if two such patients are to be operated concurrently. Only positive or suspected patients are operated in that OT. The movement corridor has been defined. The patients are not kept for observation in pre- or postoperative holding area. They are directly shifted to OT and back to the designated ward/ HDU/ ICU via designated lift after surgery. If they require immediate postoperative observation, it is being done in operation theatre itself. Patient and staff movement is minimized in the corridor. The movement corridor is sanitized after each patient movement.

Perioperative Management: The choice of anesthesia being administered, whether regional or general, depends on as per merit of the surgery; presence or absence of comorbidities and patient's choice. For proven COVID-19 negative patient, PPE by anesthesia team includes Cap, N95 mask, face shield or goggles, full sleeve gown with single use plastic apron under it and double gloves. While for surgical team it includes facial cover with hood, N95 mask, goggles, full sleeve gown with single use plastic apron under it, double gloves and shoe cover. The patient is transferred to OT with a 3-ply surgical face mask (Figure 1).

For suspected or positive patient, PPE donned by anesthesia team includes a coverall HAZMAT suit, hood cap, N95, mask, face shield and double gloves. For surgical team includes a coverall HAZMAT suit, and sterile disposable gown over it; N95, mask, face shield and double gloves. The patient is transferred to OT with a N95 face mask.

For general anesthesia, all patients are being intubated after

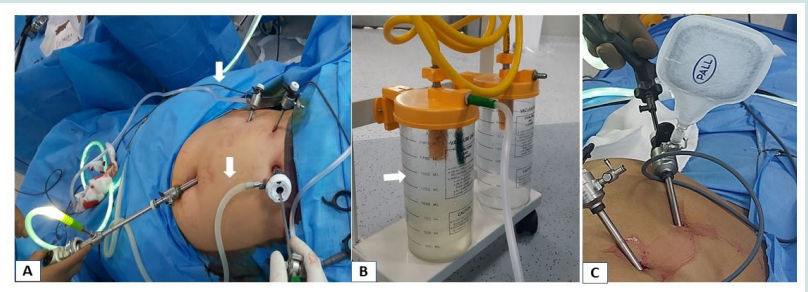

Figure 3: A - Two suction ports deployed (both depicted by arrows). B Underwater seal suction (arrow) through $0.1 \%$ sodium hypochlorite solution for smoke evacuation. $\mathrm{C}-\mathrm{Smoke}$ filter. 
Citation: Verma N, Sehgal L, Wadhawan R, Minhas V, Gupta M. General Surgery during Pandemic Era - Evolving Strategies: A Cohort Study from a Tertiary Care Centre in North India. J Surgery. 2021;9(1): 6.

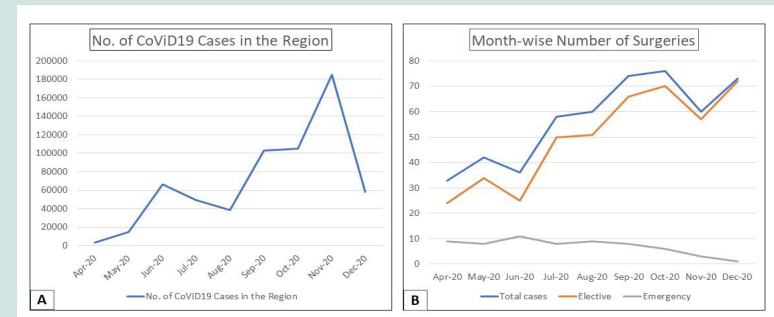

Figure 4: Comparison of month-wise trend of CoViD19 positive cases in the region and number of general surgery cases. A - Month-wise CoViD19 positive cases in the region. $\mathrm{B}$ - Month-wise general surgery cases.

rapid sequence induction and intubation. All intubations are being performed utilizing video laryngoscope (C-MAC) (Figure 2A). The patient's face along with upper chest is covered with transparent disposable plastic sheet before pre-oxygenation after due explanation to the patient (Figure 2B). The face covering sheet is removed only if there is a requirement for the surgical access. Otherwise, it is kept over the face and upper chest throughout the procedure till extubation. (Figure 2C)The sheet is removed only after ensuring safe extubation.

The surgical skin preparation includes povidone iodine followed by $2 \% \mathrm{w} / \mathrm{v}$ chlorhexidine in $70 \%$ alcohol. In all laparoscopic procedures, Veress needle is used to create pneumoperitoneum. The gas flow rates are kept low i.e. intra-abdominal pressure at $12 \mathrm{mmHg}$ and flow rate at $10 \mathrm{~L} / \mathrm{min}$ for most of laparoscopic surgeries. The settings of electrocautery are also kept at lower level 25-30 ESU (Electrosurgical unit). Two suction tubings are utilized for laparoscopic surgeries. (Figure 3A) One tube is connected from the least dependent port which evacuates smoke to the sodium hypochlorite solution in an underwater seal which is connected to the central suction. Other suction tube is connected to the suction and irrigation cannula which is used to aspirate fluid intraperitoneally during surgeries. (Figure 3B) Once available at our institution, a smoke absorbent filter (laparoshield") is also being used. (Figure 3C) Active emphasis is given at each step to reduce surgical time and reduce exposure time, wherever feasible.

\section{Training and simulation}

After approval of SOPs training and simulation exercises were conducted for all involved in patient care to ensure appropriate implementation of all safety standards.

\section{Staff Screening}

Thermal screening is done for all the staff daily. Along with this self-reporting of symptoms is encouraged. If required, testing for the presence of SARS nCov-2 via RT-PCR is done. The healthcare workers are advised isolation while awaiting report and/ or symptoms subsiding.

\section{Data Collection}

The data has been collected both retrospective and prospectively for nine months between April1, 2020 till December 31, 2020. The details recorded were demographic profile, type of surgical procedures, status for testing for RT-PCR/ CBNAAT, postoperative complications, morbidity and mortality. The statistical analysis was done using Microsoft excel.
The study was planned and duly approved by institute ethics committee. The work has been reported in line with the STROCSS criteria [7].

\section{Results}

We analysed our prospectively maintained data for 9 months (1st April 2020 to 31st December 2020). A total of 506 patients underwent 512 surgical procedures. Out of these, 446 were elective and 60 patients were admitted for Surgical emergencies. A comparison of month-wise CoViD19 positive cases in the authors' region and general surgeries (elective and emergency) performed is depicted in Figure 4 . The patient characteristics are mentioned in Table 1. It was ensured that patients were mobilised and discharged early with the mean hospital stay of 2.22 (range 1-31) days.

The details of diseases requiring surgical procedures is listed in Table 2. Emergency procedures were done for acute intestinal obstruction, Intestinal perforations, abscesses.

The surgical procedures performed during this period are listed in Table 3. 341 patients underwent Laparoscopic Surgery and 165 open surgeries. Laparoscopic cholecystectomy (39.5\%) was the most commonly performed procedure. $19.3 \%$ patients (100 Patients) underwent hernia surgery, Out of which $18 \%$ patients had laparoscopic ventral hernia repair. 2 patients (2\%) with large incisional hernia with a large defect size underwent posterior component separation with a bilateral Transversus abdominis release. 3 patients(3\%) underwent Open Overlay repair.77 patients(77\%) had groin hernia repair (58 laparoscopic and 19 open repair). Emergency laparotomy was performed for 30 patients $(5.8 \%)$ who included 23 cases of acute intestinal obstructions. One patient underwent emergency laparotomy for mesenteric artery ischemia. Re-exploration was done in this patient due to progressive gangrene of small intestine however; he later died of sepsis and multiorgan failure. 23 patients (4.4\%) underwent advanced Laparoscopic procedures, which includes 5 cases of hiatus hernia repair with laparoscopic Nissen's fundoplication 1 case of Robotic hiatus hernia repair, laparoscopic anterior resection for Carcinoma rectum (one case), Laparoscopic Right hemicolectomy for Ca Ascending colon, laparoscopic bariatric surgery for morbid obesity (13 case) which include sleeve gastrectomy, One anastomosis gastric bypass(OAGB), Roux-en-Y gastric bypass(RYGB) as well as Revision cases, 2 cases of laparoscopic heller's cardiomyotomy, laparoscopic resection of neuroendocrine tumour in first part of duodenum with Gastrojejunostomy (1 case), Laparoscopic CBD exploration for impacted stone after failed ERCP. Ileostomy closure was done in 6 cases $(1.15 \%)$ out of which one patient developed

Table 1: Patient Characteristics.

\begin{tabular}{|c|c|}
\hline Characteristics & Values/ Numbers \\
\hline Age in Years (mean $\pm \mathrm{SD}^{\#}$ ) (range) & $45.33 \pm 17.93(8-87)$ \\
\hline Weight in Kg (mean \pm SD) (range) & $67.60 \pm 10.56(15-136)$ \\
\hline Sex - males/females & $316 / 190$ \\
\hline & $\mathrm{I}-76$ \\
ASA $^{*}$ Physical Status & II -328 \\
& $\mathrm{III}-75$ \\
\hline Elective/ Emergency & $449 / 63$ \\
\hline Duration of surgery in minutes (mean \pm SD) (range) & $54.8 \pm 29.5(15-180)$ \\
\hline
\end{tabular}

\#SD - standard deviation; *ASA-American Society of Anesthesiology 
Citation: Verma N, Sehgal L, Wadhawan R, Minhas V, Gupta M. General Surgery during Pandemic Era - Evolving Strategies: A Cohort Study from a Tertiary Care Centre in North India. J Surgery. 2021;9(1): 6.

ISSN: 2332-4139

Table 2: Diagnosis at Admission.

\begin{tabular}{|c|c|}
\hline Diagnosis & Numbers $($ Total $=\mathbf{5 1 2})$ \\
\hline Cholelithiasis/ cholecystitis & 200 plus 4 pts with other combined procedures \\
\hline Hernias & 100 (76 lap + 24 open) \\
\hline Abscess/ tissue infection & 25 \\
\hline Appendicitis & 31 \\
\hline Intestinal obstruction/ perforation/Other abdominal disease requiring laparotomy & 30 \\
\hline Hemorrhoids/ fissure /fistula & 50 \\
\hline Stoma closure & 6 \\
\hline Carcinoma colon/rectum & 4 \\
\hline Hiatus hernia & 6(5 laparoscopic and 1 robotic) \\
\hline Morbid obesity & 13 ( 11 laparoscopic and 2 endoscopic) \\
\hline Duodenum first part tumour & 1 \\
\hline Varicocele & 2 \\
\hline Achalasia Cardia & 2 \\
\hline Choledocholithiasis & 1 \\
\hline Varicose viens & 2 \\
\hline Soft tissue excision & 20 \\
\hline Phimosis & 2 \\
\hline Hydrocele & 1 \\
\hline Suspected Ca Testis/ testicular necrosis & 3 \\
\hline Lacerated wound & 2 \\
\hline
\end{tabular}

Table 3: List of Surgical Procedures.

\begin{tabular}{|c|c|}
\hline Procedures & Numbers $($ Total $=512)$ \\
\hline Laparoscopic/Robotic & 338 \\
\hline Cholecystectomy & 204 \\
\hline Appendectomy & 31 \\
\hline Hernioplasty & 76 \\
\hline Laparoscopic / endoscopic bariatric procedures & 13 \\
\hline Laparoscopic/Robotic Upper GI procedures(Hiatus/achalasia) & 8 \\
\hline Other laparoscopic procedures & 4 \\
\hline Open/ other approaches & 168 \\
\hline Debridement/ Incision and drainage & 25 \\
\hline Laparotomy & 30 \\
\hline Hernioplasty & 24 \\
\hline Ileostomy closure & 6 \\
\hline Other open procedures & 29 \\
\hline
\end{tabular}

anastomotic leak due to previous adhesions, re-exploration and revision of stoma was done. One patient underwent an open anterior resection with a proximal ileostomy for a carcinoma rectosigmoid presenting with intestinal obstruction.

489 Patients were tested preoperatively for SARS nCov-2 by the RT-PCR, 6 patients were tested by CBNAAT who were admitted via emergency and early report was required. Remaining 11 patients were clinically and radiologically screened for COVID-19 as per government and authors' hospital guidelines which was during initial 10 days of study as RT-PCR was not available at that time. Xray-chest was also done for all patients to assist diagnosis in addition along with a detailed questionnaire, clinical and family history. In one patient for elective surgery, High resolution computed tomography scan for chest was done for infiltrates on X-ray chest. The RT-PCR for SARS nCov2 was negative for this patient. 22 patients who were planned for elective surgeries were found to be COVID positive so Surgeries were deferred for at least three weeks and they were operated later after full recovery and negative report.

Two of co-authors developed symptoms of COVID-19 and tested positive for SARS nCov-2 in July 2020.

\section{Discussion}

With the occurrence COVID19 pandemic, the patient care has been challenging. The provider and patient safety both have to be ensured along with smooth conduct of the procedure. Many healthcare organizations have refrained from and advised against 
conducting elective surgical procedures. First, there is enhanced risk of infection to healthcare workers due to increased aerosol generation in operation theatre [8]. Second, it may to ensure optimal resource allocation (manpower, PPE and other consumables) to sick patients suffering from Covid-19. Third, there could be fear in the public visiting healthcare setting and risk of acquiring Covid 19 infection [9]. However, the number of elective cases has been showing uptrend except for November 2020 despite high number of CoVid19 positive cases in the region (Figure 4). This could have happened as with each month; the healthcare systems generated more confidence among public with regard to safety for elective procedures. Additionally, after a long waiting, the patients were now possibly unwilling for further delay in elective procedures.

There are various challenges encountered at authors' institute as well towards that. It has been considered ideal to have a negative pressure operation theatre to contain the infection [10]. However, it may not be feasible. There is a complex comprising of 10 OTs at authors' hospital where majority of procedures are conducted. There is another OT attached with labour room and neonatal intensive care unit. The possibility of creating a negative pressure OT was explored at our hospital. But it was rendered not feasible by the engineering department. Labour room complex OT was also considered for conducting surgeries for suspected/ positive patients. However, it would have interfered with routine activity of labour room and NICU. Hence, an OT was dedicated with in main OT complex for performing procedures on suspected/ positive patients along with a backup plan and a designated movement corridor. More emphasis was given on training and simulations to ensure safety. This included repeated training, simulation of patient movement in corridors and care in OT utilizing dummies. Along with that feedbacks received during the training were noted and if and the editing was done in training modules accordingly. In addition, the training and simulation were recorded and reviewed and gaps were identified and modified. Further, the videos have been utilized for training the next batches and for the refresher. Tong et al have reported managing their OTs for CoVid19 positive patients without having a negative pressure OT. They emphasized on the importance of repeated training and simulations [11].

Maintaining social distancing in hospitals may not be easy. And it is infeasible to test every patient visiting OPD [12]. Video consultation is a solution for non-emergency disease which can be postponed and dealt electively after some time [13]. It ensures care while maintaining social distancing. Further, contact time is minimized at subsequent OPD visits.

Getting every patient undergoing procedure tested for SARS nCov2 has been also a challenge. It has been due to availability of testing kits and change in government policies on testing with gain of further knowledge regarding virus and disease. Further, the problem could be addressed differently by different state governments as health is the responsibility of state government [14]. Hence all patients undergoing elective/ emergency procedure could not be tested initially. However, later on testing became a norm for all patients once more kits and options have been available. The availability of both RT-PCR and CBNAAT at authors institute has offered more flexibility and lead to judicious resource utilization. Further, testing for all patients for elective procedures before planned admission in last three months has been able to add to safety and avoid undue hospital admission for the patients who tested positive for SARS nCov-2.

In all patients, emphasis on additional clinical evaluation for the presence of any signs and symptoms related to CoViD19 was given. The risk of morbidity and mortality is quite high among patients undergoing elective surgeries in CoViD19 positive patients [15]. However, RT-PCR has moderate sensitivity [16]. In case of high suspicion, a repeat testing and CT scanning of chest is being done at authors' institute. The authors recommend testing for all patients before elective and urgent procedures.

The laparoscopic guided procedures have distinct advantages. These include reduced pain, morbidity and length of stay for patients. It could also mean easier access for the operating surgeons. However, laparoscopic procedures have been perceived with higher risk of aerosol generation and thereby increased risk of exposure to healthcare workers [5]. While laparoscopic procedures have not been found to be associated with increased risk by others[17]. The authors instituted various measures to reduce the risk of aerosol generation and exposure. Utilization of two suctions has been one among them. Here one suction port has been designated for gas and smoke evacuation through an underwater seal in $0.1 \%$ sodium hypochlorite solution. The solution has been shown to reduce corona virus infectivity within one minute [18]. Other measures included utilization lower pressure and flow rates, complete gas and smoke evacuation through suction and utilization of smoke filters. [18-20]

Similarly, airway management has been perceived with higher risk of aerosol exposure. Various measures have been adopted for risk reduction. These include utilization of transparent sheet covering, video laryngoscopy, rapid sequence induction-intubation and ensuring safe extubation by avoiding coughing and bucking over endotracheal tube.

\section{Conclusions}

COVID pandemic continues to be a major challenge in front of world. The pandemic may last for a longer period and healthcare workers are also infected very frequently. Proper screening and testing of patient as well as healthcare workers is of utmost importance to prevent chain of spread in hospital and healthcare workers. Utilization of appropriate PPE, technique of induction and extubation; least amount of gas release, proper evacuation of smoke/ gas, reduction of exposure time are important steps to carry out both elective and emergency procedures safely. Various guidelines have been issued by different academic associations. However, every institution has to improvise depending upon the local resources and infrastructure available. Further, with continuing pandemic, it will be prudent that every institution creates infrastructural changes to cater continuous inflow of patients over a longer period.

\section{References}

1. Report of Clustering Pneumonia of Unknown Etiology in Wuhan City. Wuhan Municipal Health Commission 2019.

2. COVID-19: Global tally, death toll peak in December.

3. National Coronavirus update 2020.

4. Kupferschmidt K (2021) New Coronavirus variants could cause more 
Citation: Verma N, Sehgal L, Wadhawan R, Minhas V, Gupta M. General Surgery during Pandemic Era - Evolving Strategies: A Cohort Study from a Tertiary Care Centre in North India. J Surgery. 2021;9(1): 6.

ISSN: $2332-4139$

reinfections, require updated vaccines

5. Johnson G K, Robinson W S (1991) Human immunodeficiency virus-1 (HIV1 ) in the vapors of surgical power instruments $33: 47-50$

6. Gloster HM, Roenigk RK (1995) Risk of acquiring human papillomavirus from the plume produced by the carbon dioxide laser in the treatment of warts. $J$ Am Acad Dermatol 32: 436-441.

7. Agha R, Abdall-Razak A, Crossley E, Dowlut N, losifidis C, et al. (2019) for the STROCSS Group. The STROCSS 2019 Guideline: Strengthening the Reporting of Cohort Studies in Surgery. Int J Surg . 72:156-165.

8. Johnson G K, Robinson W S (1991) Human immunodeficiency virus-1 (HIV1 ) in the vapors of surgical power instruments $33: 47-50$

9. Protocols for elective surgeries during COVID-19-Express Healthcare,

10. Brindle ME, Gawande A (2020) Managing COVID-19 in surgical systems. Ann Surg 272: 1-2.

11. Tong QJ, Chai JX, Tan LH, Prit S, Ong LT, et al.(2020) Assessing Operating Room Preparedness for COVID-19 Patients through In-Situ Simulations. Anesthesia \& Analgesia: 131: 104-106.

12. Centers for Disease Control and Prevention. Interim guidance for healthcare facilities: preparing for community transmission of COVID-19 in the United States.
13. Centers for Disease Control and Prevention. Interim infection prevention and control recommendations for patients with suspected or confirmed coronavirus disease 2019 (COVID-19) in healthcare settings.

14. Schreiner M (2016) Simple Poverty Scorecard Poverty-Assessment Tool: India. Simple Poverty Score Card.

15. American College of Surgeons (2020) COVID-19: Guidance for triage of nonemergent surgical procedures.

16. Francis N, Dort J, Cho E, Feldman L, Keller D, et al. (2020) SAGES and EAES recommendations for minimally invasive surgery during COVID-19 pandemic, Surg. Endosc. 34: 2327-2331.

17. Pryor A (2020) SAGES and EAES recommendations regarding surgical response to COVID-19 crisis.

18. AMASI (Association of minimal Access Surgeons of India) (2020) Guidelines for Conducting Minimal Access Surgery during COVID-19 Pandemic.

19. Veziant J, Bourdel N, Slim K (2020) Risks of viral contamination in healthcare professionals during laparoscopy in the Covid-19 pandemic. Journal of Visceral Surgery 157: 59-62.

20. IAGES (Indian Association of Gastrointestinal Endoscopic Surgeons) (2020) Covid Surgery Recommendations. 